Article

\title{
Improving the Efficiency of Robot-Mediated Rehabilitation by Using a New Organizational Model: An Observational Feasibility Study in an Italian Rehabilitation Center
}

\author{
Irene Aprile ${ }^{1}$, Cristiano Pecchioli ${ }^{1}$, Simona Loreti ${ }^{1}$, Arianna Cruciani ${ }^{1}$, Luca Padua ${ }^{2,3}$ and \\ Marco Germanotta ${ }^{4, *}$ i \\ 1 IRCCS Fondazione Don Carlo Gnocchi, 20121 Milan, Italy; iaprile@dongnocchi.it (I.A.); \\ cpecchioli@dongnocchi.it (C.P.); sim.lor@tiscali.it (S.L.); ariannacrux@gmail.com (A.C.) \\ 2 Department of Geriatrics, Neurosciences and Orthopaedics, Università Cattolica del Sacro Cuore, \\ 00168 Rome, Italy; luca.padua@unicatt.it \\ 3 Fondazione Policlinico Universitario Agostino Gemelli IRCSS, 00168 Rome, Italy \\ 4 IRCCS Fondazione Don Carlo Gnocchi, 50143 Florence, Italy \\ * Correspondence: mgermanotta@dongnocchi.it; Tel.: +39-06-3308-6448
}

Received: 28 October 2019; Accepted: 4 December 2019; Published: 8 December 2019

Featured Application: By using new organizational models, robotic rehabilitation can be sustainable; therefore, it is possible to provide high technological upper limb treatment to a high number of patients.

\begin{abstract}
Robotic rehabilitation is a promising way to restore upper limb function, but new organizational models are crucial in order to improve its sustainability. We aimed to analyze the feasibility of a robot-Assisted REhabilitation Area (robotAREA) equipped with four devices, using a new organizational model in which one physiotherapist supervises up to four patients. In 33 days, 60 patients were treated in the robotAREA. Two hundred fifty-five rehabilitation sessions were analyzed, each including two, three, or four patients supervised by one physiotherapist (the R2, R3, and R4 group, respectively). For each session, the duration of (a) setup, (b) training, (c) supervision, (d) session closure, and participant satisfaction were measured. For each patient, upper limb impairment, disability, mobility, and comorbidity were recorded. The time of training in the R4 group was lower by only 2 and 3 min compared to the R2 and R3 groups, respectively, while the supervision significantly changed between the R2, R3, and R4 groups. No differences were observed in satisfaction. Moreover, according to the logistic regression analysis, when the mean disability of the group is moderate, four patients can be supervised by one physiotherapist. These results suggest that the proposed model is feasible, and the mean disability of the group could influence the proper physiotherapist/patient ratio.
\end{abstract}

Keywords: rehabilitation; robotics; technological devices; upper limb impairment; organizational model

\section{Introduction}

Upper limb impairment is defined by the International Classification of Functioning, Disability and Health model [1] as (1) impairments of body function, such as a significant deviation or loss in neuromusculoskeletal and movement-related function related to joint mobility, muscle power, muscle tone, and/or involuntary movements, or (2) impairment of body structures, such as a significant deviation in structure of the nervous system or structures related to movement. 
Robot-mediated therapy after upper limb impairment, because of neurological or orthopedic diseases, is emerging in the rehabilitation field. A recent Cochrane review highlighted a potential benefit of the electromechanical and robot-assisted upper limb training in stroke patients [2]. In addition, robotic therapy can increase the efficiency of therapy, and therefore the accessibility to treatment [3]. In fact, in an era of increasing focus on healthcare resource utilization, a considerable interest is devoted toward reducing costs while preserving a high-quality level. From this perspective, economic evaluations of rehabilitation interventions using cost-effectiveness and/or cost-utility analysis are needed [4]. However, few studies have been conducted on the real costs of robotic and technological rehabilitation when compared to traditional rehabilitation. Wagner et al. [5] found that the costs of an additional upper limb treatment (robotic or conventional) in stroke patients can be recuperated because of lower general healthcare costs. Moreover, a recent systematic review, including five studies, found that robotic therapy produces a better economic outcome when compared to conventional therapy [6].

In addition, Masiero et al. [7] suggested that the availability of a room equipped with more than one device can further increase the sustainability of the treatment, addressing an essential need of under-resourced healthcare systems [8]. In fact, although robotic devices are expensive, new organizational models where one physiotherapist supervises more than one patient could optimize the human resources. Some authors [9-11] suggested that this approach can be as effective as traditional therapy for stroke patients, presenting a more cost- and labor-efficient option. However, to the best of our knowledge, there have been no previous studies that analyzed either the rehabilitation dose provided to the patients or patient's satisfaction when different physiotherapist/patient ratios have been used. Moreover, the clinical characteristics of patients that could influence the proper physiotherapist/patient ratio remain uninvestigated.

In April 2016, to implement the robotic rehabilitation in a sustainable way, a set of four technological and robotic devices for the upper limb was identified, and a robotic rehabilitation area was built and equipped with the set [12]. This allowed experimenting with a new organizational model where more than one patient is supervised by one physiotherapist, in order to increase the efficiency of the robotic treatment.

Then, to critically evaluate the feasibility of this model, a study based on data related to the use of these devices in clinical practice was carried out.

Specifically, the aim of this study was twofold: (1) to verify the feasibility, in terms of rehabilitation dose (as measured by the time of training) and patient satisfaction (as measured by the Visual Analogue Scale for Satisfaction), of a new organizational model in which one physiotherapist supervises up to four patients using a set of four robotic-technological devices; and (2) to identify the optimal number of patients that can be supervised by one physiotherapist, in accordance to the patients' upper limb impairment (measured by the Motricity Index), disability (measured by the Barthel Index), mobility (measured by the Deambulation Index), and comorbidity (measured by the Cumulative Illness Rating Scale).

\section{Materials and Methods}

\subsection{Study Design}

An observational feasibility study examining the daily activity of the robot-Assisted REhabilitation Area (robotAREA) of a rehabilitation center in Italy.

\subsection{Ethics}

The study was carried out in compliance with the Helsinki Declaration. All the patients (both inpatients and outpatients) provided signed written informed consent to use collected data for clinical research purposes at the admission in the rehabilitation center. This study was approved by the Ethics and Experimental Research Committee of the "IRCCS Fondazione Don Carlo Gnocchi" (Prot. N.8/2016CE_FDG/FC/SA) on 6 April 2016. 


\subsection{Setting}

The study was conducted over a period of 33 days (from February to March 2017). The RobotAREA is equipped with a set of four devices, three robots (MOTORE, Humanware, Italy, and Amadeo and Diego, Tyromotion, Austria), and one sensor-based device (Pablo, Tyromotion, Austria) [12]. MOTORE is a robotic device that allows passive, active, and active-assistive planar movements of the shoulder and elbow joints; Amadeo is a robotic device that allows passive, active, and active-assistive flexion and extension finger movements (also separately for each finger); Diego is a robotic device that allows three-dimensional, unimanual, and bimanual movements of the shoulder joint, both in sitting and in standing, with gravity compensation; Pablo is a sensor-based device that requires patients to perform unassisted three-dimensional movements of the shoulder, elbow, and wrist joints, both unimanual and bimanual, both when sitting and standing. All physiotherapists who worked in the RobotAREA had an expertise in robotic rehabilitation and used these devices for more than one year. Figure 1 shows the distribution of the device stations inside the robotic-technological area.

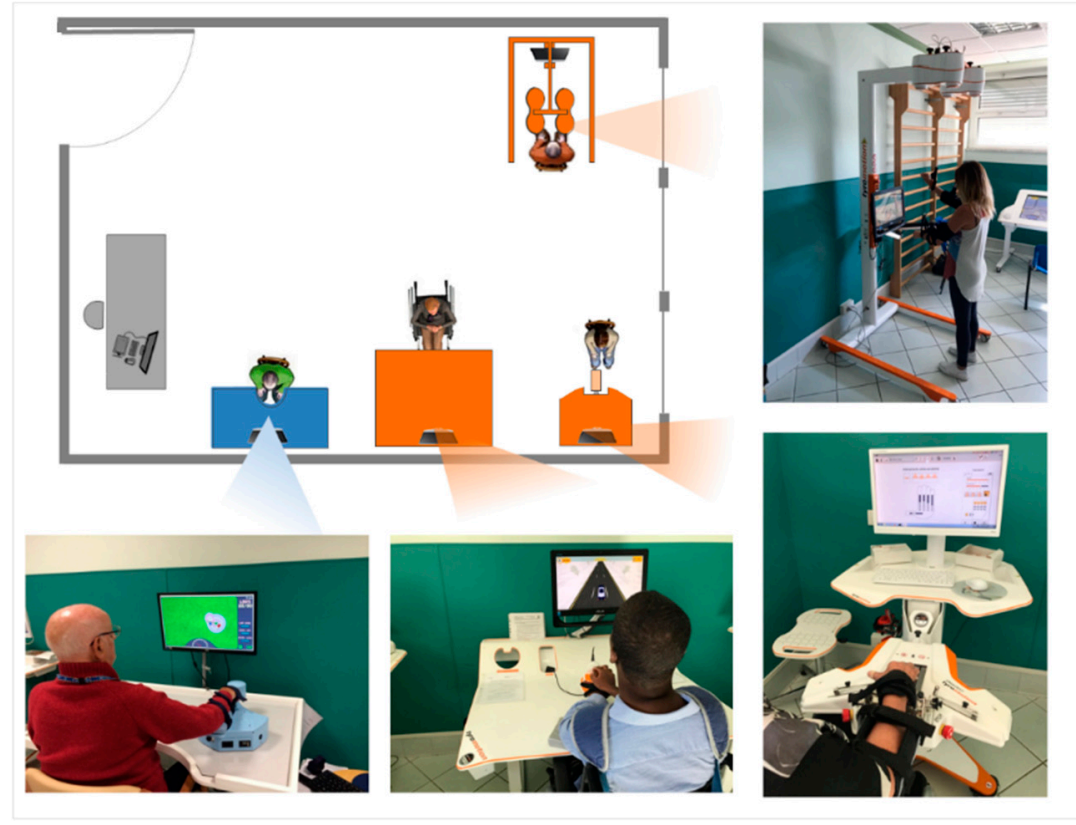

Figure 1. Distribution of the devices in the robot-Assisted REhabilitation Area (robotAREA). Beginning by the door, clockwise: Diego; Amadeo; Pablo; MOTORE.

\subsection{Participants}

Inclusion criteria: both inpatients and outpatients admitted to our rehabilitation facility with an upper limb impairment due to neurologic or orthopedic disorders. We excluded patients with a contraindication to robotic therapy, which was identified as: (1) inability to understand the physiotherapist's instructions and device exercises; (2) fixed contraction deformity/excessive spasticity; (3) any psychiatric disorder or severe cognitive disturbance that could reduce treatment compliance; and/or (4) severe visual impairment.

\subsection{Robotic Rehabilitation Treatment}

Each RS lasted $45 \mathrm{~min}$, and the training frequency was 3 or 5 times/week, depending on the rehabilitation setting (outpatients or inpatients, respectively). During the treatment, both the distal and the proximal segments of the patients' upper arm were treated by means of the robotic and sensor-based devices. Motor and cognitive tasks were performed during the treatment, while visual and auditory feedback was provided to help the patients. During each session, the physical therapist uses one system for each patient to minimize the time required to move the patients from one system 
to another. The rehabilitation program starts with the robotic device for the shoulder and elbow joints, followed by the robotic device for the hand, the sensor-based device for the shoulder, elbow, and wrist and, finally, the robotic system for the shoulder. The adopted protocol followed the general indications, which were laid out in a flow chart, in order to ensure the homogeneity of treatment; however, for each patient, the physiotherapist selected the device and adapted the exercises to his/her residual abilities. More details on the rehabilitation protocol were previously reported [13]. During the rehabilitation session (RS), one physiotherapist supervised more patients at once with a physiotherapist/patient ratio of $1 / 2$ (R2), 1/3 (R3), or $1 / 4$ (R4). The number of patients treated in each RS was chosen by the physician depending on the upper limb impairment, disability, mobility, and comorbidity of the entire group, based on the experience done with a wide multicenter study [13].

\subsection{Data Collection}

A trained researcher monitored the patients during each robotic-technological rehabilitation session (RS). The data collected during each session for each patient was called "observation". During the study, for each patient, several observations were performed, according to the individual rehabilitation program. In order to limit bias due to researcher's presence, neither physiotherapists nor patients knew when data collection would take place.

To investigate whether and in which patients this new organizational model was applicable, we compared the different rehabilitation settings (R2, R3, and R4) in terms of the rehabilitation dose and clinical characteristics of the patients. For each session, the number of patients supervised by one physiotherapist was recorded, together with the clinical status of the patients, using validated clinical scales administered by trained operators. Specifically, we evaluated disability (according to the Barthel Index, BI [14]), upper limb impairment (using the Motricity Index, MI [15]), mobility (according to the Deambulation Index, DI [16]), comorbidities and severity (described according to the Cumulative Illness Rating Scale-Comorbidity Index, CIRS-CI, and the Cumulative Illness Rating Scale-Severity Index, CIRS-SI [17-19]). Complete demographic and clinical details were collected at the first observation for each patient.

Moreover, to evaluate the feasibility of the model, the following measures were recorded: (a) tSetup (minutes): time spent for the correct patient placement and for planning of the session exercises; (b) tTraining (minutes): time spent by patient to perform the training; (c) tSupervision (minutes): time spent by the physiotherapist in the individual interaction with the patient; and (d) tClosure (minutes): time spent by the physiotherapist to close the exercise session and remove the patient from the device. These measures were obtained in a subsample of observations. In addition, the Visual Analogue Scale for Satisfaction [20], a self-assessment scale ranging from 0 (no satisfaction) to 10 (extreme satisfaction), was used to measure patients' satisfaction. The exact question was "Are you satisfied with your rehabilitation session?"

\subsection{Statistical Analysis}

To evaluate the feasibility of the model, data obtained from the three groups were compared by using one-way ANOVA tests, followed by post-hoc analysis with Bonferroni correction, or Kruskal-Wallis tests, followed by post-hoc analysis, using Dunn's procedure with a Bonferroni correction, as appropriated.

To evaluate the impact of disability, upper limb impairment, mobility, and comorbidity on the number of patients in each group, for each session, the mean data of the group were computed and compared between groups (univariate analysis) using Kruskal-Wallis tests, followed by post-hoc analysis, using Dunn's procedure with a Bonferroni correction. Then, to identify the best physiotherapist/patient ratio, a multivariate logistic analysis was performed, considering the variables identified in the univariate analysis as covariates. Finally, a receiver operating characteristics ROC analysis was used to identify a possible cutoff. 
For all statistical tests, the significance level was set to 0.05 . Statistical analysis was performed using SPSS (IBM Corp. Released 2017. IBM SPSS Statistics for Windows, Version 25.0. Armonk, NY, USA: IBM Corp.).

\section{Results}

In a period of 33 days, 60 patients were observed in our rehabilitation department. Table 1 shows the demographic and clinical characteristics of the analyzed sample. Spinal disorders included spinal cord injury after trauma or surgery on the column for neoplasia/lumbar spinal stenosis or spinal disc herniation; orthopedic disease included patients with shoulder prosthesis, upper limb fractures/surgery, and polytrauma. Upper limb impairment, disability, mobility, and comorbidities are reported in Table 2 . During the study, each patient performed a variable number of rehabilitation sessions depending on the hospitalization stay and frequency of treatment per week. No serious adverse events were observed, and the robotic therapy was well accepted by all patients, without changes in the initial plan. None of the patients declined to participate in the study, since it did not interfere with their rehabilitation plan.

Table 1. Demographic and clinical characteristics of the observed sample.

\begin{tabular}{ccc}
\hline Age (Years) & \multicolumn{2}{c}{$\mathbf{6 4} \pm \mathbf{1 6}$ (Range: 19-91) } \\
\hline \multirow{2}{*}{ Sex } & Male & $27(45.0 \%)$ \\
& Female & $33(55.0 \%)$ \\
\hline \multirow{2}{*}{ Setting } & Outpatient & $11(18.3 \%)$ \\
& Inpatient & $49(81.7 \%)$ \\
\hline \multirow{2}{*}{ Diagnosis } & Stroke & $41(68.3 \%)$ \\
& Multiple sclerosis & $3(5.0 \%)$ \\
& Charcot Marie Tooth & $1(1.7 \%)$ \\
& Guillain Barrè syndrome & $1(1.7 \%)$ \\
& Motoneuron disease & $1(1.7 \%)$ \\
& Parkinson disease & $1(1.7 \%)$ \\
& Spinal disorder & $7(11.7 \%)$ \\
& Orthopedic disease/trauma & $5(8.3 \%)$ \\
\hline
\end{tabular}

Table 2. Disability, upper limb impairment, mobility, and comorbidities of the sample.

\begin{tabular}{lcc}
\hline Variable & Mean \pm SD & Range \\
\hline Barthel Index & $57.7 \pm 23.1$ & $(14-97)$ \\
Motricity Index & $57.5 \pm 23.2$ & $(1-93)$ \\
Deambulation Index & $2.9 \pm 2.2$ & $(0-7)$ \\
CIRS-SI & $1.0 \pm 0.4$ & $(0.3-2)$ \\
CIRS-CI & $1.9 \pm 1.0$ & $(0-6)$ \\
\hline
\end{tabular}

SD: standard deviation; CIRS-CI: Cumulative Illness Rating Scale-Comorbidity Index; CIRS-SI: Cumulative Illness Rating Scale-Severity Index.

The total observations (data collected at each session for each patient) were 782, with a mean of 24 observations/day (range: 17-31) and a mean of 13 observations/patient (range: 1-50) (see also the supporting document: Supplementary Table S1). Two hundred fifty-five rehabilitation sessions were recorded with a ratio of physiotherapist/patient as follows: 45 sessions $(17.7 \%)$ with a ratio of $1 / 2$ (R2); 148 sessions $(58.0 \%)$ with a ratio of $1 / 3(\mathrm{R} 3)$, and 62 sessions (24.3\%) with a ratio of $1 / 4(\mathrm{R} 4)$. The mean value of the physiotherapist/patient ratio was $1 / 3.1$.

\subsection{Feasibility of the Model}

Data related to the rehabilitation dose, supervision, and patients' satisfaction were recorded in a subsample of 329 observations (supporting document: Supplementary Table S1) and reported in 
Table 3. As expected, the tTraining was significantly different between groups $(p=0.007)$; post-hoc analysis showed that it was significantly lower in the R4 group, when compared with both R2 (mean difference: $3.2 \mathrm{~min}$ ) and R3 groups (mean difference: $2.3 \mathrm{~min}$ ). Instead, no differences in tTraining were found between R2 and R3 groups. The tSupervision was significantly different between all groups $(p<0.001)$, as confirmed also by post-hoc analysis. With respect to patient satisfaction, no significant differences were observed between groups $(p=0.39)$.

Table 3. Feasibility measurements: rehabilitation dose (tTraining) and supervision time (tSupervision) during a 45-min rehabilitation session, and patients' satisfaction in the whole sample and the three groups, separately, together with the statistical analysis. Data were obtained from 329 observations.

\begin{tabular}{|c|c|c|c|c|c|c|c|c|}
\hline & \multirow{2}{*}{$\begin{array}{c}\text { Whole Group } \\
\text { Mean } \pm \text { SD }\end{array}$} & \multirow{2}{*}{$\begin{array}{c}\text { R2 } \\
\text { Mean } \pm S D\end{array}$} & \multirow{2}{*}{$\begin{array}{c}\mathrm{R} 3 \\
\text { Mean } \pm \mathrm{SD}\end{array}$} & \multirow{2}{*}{$\begin{array}{c}\mathbf{R} 4 \\
\text { Mean } \pm \text { SD }\end{array}$} & \multirow{2}{*}{$p$} & \multicolumn{3}{|c|}{ Post-Hoc Analysis } \\
\hline & & & & & & R2 vs. R3 & R2 vs. R4 & R3 vs. R4 \\
\hline tTraining (min) & $31.3 \pm 6.4$ & $32.8 \pm 5.0$ & $31.9 \pm 6.0$ & $29.6 \pm 7.2$ & 0.007 & $>0.99$ & 0.04 & 0.01 \\
\hline $\begin{array}{l}\text { tSupervision } \\
\text { (min) }\end{array}$ & $9.7 \pm 2.3$ & $11.0 \pm 3.2$ & $9.7 \pm 2.0$ & $8.9 \pm 2.1$ & $<0.001$ & 0.005 & $<0.001$ & 0.007 \\
\hline $\begin{array}{l}\text { VAS for } \\
\text { satisfaction }\end{array}$ & $8 \pm 1$ & $8 \pm 2$ & $8 \pm 1$ & $8 \pm 1$ & 0.39 & - & - & - \\
\hline
\end{tabular}

R2, R3 and R4 indicate groups with 2, 3 or 4 patients supervised by one physiotherapist, respectively. Values in bold indicate statistical significance $(p<0.05)$. SD: standard deviation; VAS: Visual analogue scale.

In addition, we found that the mean time needed for the setup (tSetup) was $3.3 \pm 1.3 \mathrm{~min}$, while the mean time needed for the session closure (tClosure) was $1.4 \pm 0.5 \mathrm{~min}$.

\subsection{Impact of Disability, Upper Limb Impairment, Mobility, and Comorbidity on the Model}

A comparison of upper limb impairment, disability, mobility, and comorbidity mean score between the three groups was made and reported in Table 4. The results showed significant differences only in BI, DI, and CIRS-SI between the groups R2 versus R4, and R3 versus R4. Considering the lack of statistical significance between the R2 and R3 groups, a further analysis was made combining the R2 and $\mathrm{R} 3$ groups (R2 + R3 group). The logistic regression model was carried out on the three statistically significant variables obtained from the univariate analysis. We found that the model was statistically significant, $\chi 2(3)=36.096, p<0.001$. As shown in Table 5, among the three predictor variables, only the BI was statistically significant. To obtain a reference value for the only significant variable (BI) in the logistic regression model, an ROC analysis was carried out. According to the Youden index, the obtained cut-off value was equal to 57.

Table 4. Disability, mobility, and comorbidity variables for the three groups, separately. Data were obtained from 782 observations.

\begin{tabular}{|c|c|c|c|c|c|c|c|}
\hline & \multirow{2}{*}{$\frac{\mathrm{R} 2}{\text { Mean } \pm \mathrm{SD}}$} & \multirow{2}{*}{$\begin{array}{c}\text { R3 } \\
\text { Mean } \pm \text { SD }\end{array}$} & \multirow{2}{*}{$\frac{\mathrm{R} 4}{\text { Mean } \pm \text { SD }}$} & \multirow{2}{*}{$p$} & \multicolumn{3}{|c|}{ Post-Hoc Analysis } \\
\hline & & & & & R2 vs. R3 & R2 vs. R4 & R3 vs. R4 \\
\hline $\mathrm{BI}$ & $49.0 \pm 10$ & $53.0 \pm 12.6$ & $63.0 \pm 14.3$ & $<0.001$ & 0.24 & $<0.001$ & $<0.001$ \\
\hline MI & $59.5 \pm 14.7$ & $57.2 \pm 11.8$ & $57.9 \pm 8.8$ & 0.42 & - & - & - \\
\hline DI & $2.1 \pm 1.2$ & $2.4 \pm 1.4$ & $3.3 \pm 1.4$ & $<0.001$ & 0.98 & $<0.001$ & $<0.001$ \\
\hline CIRS-SI & $1.1 \pm 0.3$ & $1.0 \pm 0.2$ & $0.9 \pm 0.2$ & $<0.001$ & 0.26 & $<0.001$ & $<0.001$ \\
\hline CIRS-CI & $2.1 \pm 0.8$ & $2.0 \pm 0.6$ & $1.8 \pm 0.4$ & 0.06 & - & - & - \\
\hline
\end{tabular}

Values in bold indicate statistical significance $(p<0.05)$. R2, R3 and R4 indicate groups with two, three, or four patients supervised by one physiotherapist, respectively. SD: standard deviation; BI: Barthel Index; MI: Motricity Index; DI: Deambulation Index; CIRS-CI: Cumulative Illness Rating Scale-Comorbidity Index; CIRS-SI: Cumulative Illness Rating Scale-Severity Index. 
Table 5. Logistic regression model for the identification of the best physiotherapist/patient ratio (R2/R3 versus R4), considering the mean disability, mobility, and comorbidity of the group (identified in the univariate analysis). Data were obtained from 255 rehabilitation sessions (782 observations).

\begin{tabular}{lcccccc}
\hline & $\boldsymbol{\beta}$ & $\begin{array}{c}\text { Standard } \\
\text { Error }\end{array}$ & $\boldsymbol{p}$ & \multicolumn{2}{c}{ Exp $(\boldsymbol{\beta})$} & \multicolumn{2}{c}{$\mathbf{9 5 \%}$ Confidence Interval for Exp ( $\beta)$} \\
\cline { 5 - 6 } & & & & Relative Risk & Lower Bound & Upper Bound \\
\hline BI & 0.099259 & 0.039318 & $\mathbf{0 . 0 1}$ & 1.1044 & 1.0224 & 1.1928 \\
DI & -0.52213 & 0.37 & 0.16 & 0.5933 & 0.2873 & 1.2252 \\
CIRS-SI & -1.41553 & 0.75418 & 0.06 & 0.2428 & 0.0554 & 1.0647 \\
\hline
\end{tabular}

Values in bold indicate statistical significance $(p<0.05)$. BI: Barthel Index; DI: Deambulation Index; CIRS-SI: Cumulative Illness Rating Scale-Severity Index.

\section{Discussion}

Robotic tools are emerging in the rehabilitation field, showing promising expectations for an intensive and personalized treatment. Nonetheless, these devices are expensive. Therefore, it is necessary to find the best organizational models to increase the number of patients who may have access to the technological rehabilitation, optimizing human resources. In this perspective, it is crucial to know if and how patient's disability, mobility, or comorbidity can influence an organizational model where a new, non-traditional ratio of physiotherapist/patient would be adopted.

This study shows the feasibility, in terms of rehabilitation dose and patient's satisfaction, of an organizational model with one physiotherapist supervising more than one patient, using a set of four devices for a comprehensive upper limb rehabilitation. In a 45-min rehabilitation session, regardless of the physiotherapist/patient ratio, each patient performed about $30 \mathrm{~min}$ of training with an individual physiotherapist supervision of about $10 \mathrm{~min}$. As expected, the time of training was significantly lower in the R4 group but with a gap of only 2 and 3 min compared to the R2 and R3 groups, respectively. Regarding the difference in the training time recorded between the R2/R3 and R4 groups, an increase of about $3 \mathrm{~min}$ of the rehabilitation session of the R4 group can be used to overcome this small gap.

Therefore, our results showed that the physiotherapists in this study were able to supervise up to four patients, without a reduction of the treatment dose provided to the patient. This is a crucial point: in fact, several studies report an intensity-effect relation between the amount of therapy that individuals receive and the motor recovery [21,22]; additionally, no ceiling effect for the intensity of the therapy has been observed [23-25]. Note that patient satisfaction was very high (8/10) without significant differences between groups, so patients were very satisfied even if treated together with one, two, or three other patients at the same time. Regarding the clinical characteristics of the patients, our data did not show differences when two or three patients were supervised by one physiotherapist. On the contrary, though as expected, the group in which four patients were supervised by one physiotherapist (R4) had a lower disability and comorbidity and higher mobility than the R2 and R3 groups. A remarkable result is that upper limb impairment did not influence the organizational model proposed, since the MI not significantly different between the groups (R2, R3, and R4). The multivariate analysis showed that only the BI can predict significantly the treatment group, meaning that the maximum number of patients supervised by one physiotherapist can be dependent on their level of autonomy. Therefore, the BI seems to be the most important variable that could influence the physiotherapist/patient ratio. Based on our results, a ratio of $1 / 4$ could be used if the mean value BI of the group of patients is higher than 57. Note that patients with BI values lower than 60 are considered patients with a severe disability [14]. Our results showed a similar cut-off of disability, even if calculated as the mean of the group (BI mean score of group <57).

The analysis of both clinical data and feasibility measures suggests that patients with a severe disability (BI mean score of the group $<57$ ) can be equally treated in groups of two or three patients, providing that they had the same dose. Instead, when a moderate disability is present, up to four patients can be supervised by one physiotherapist. This study also confirms the possibility of optimizing resources allocation. In fact, in our rehabilitation planning, a mean of 24 patients were treated each 
day with a mean value of physiotherapist/patient ratio of $1 / 3.1$, which means eight rehabilitation sessions/day. In a traditional rehabilitation model (with a physiotherapist/patient ratio of 1/1) to treat 24 patients/day, obviously 24 rehabilitation sessions/day are needed. As highlighted in the proposed organizational model, it is possible to reduce the number of rehabilitation sessions/day (needed to treat the same number of patients) by about $1 / 3$, with better human resources allocation.

Moreover, it is worthy to note that we have specifically chosen a set of devices with a limited time required to start the rehabilitation session, as highlighted by the reported tSetup, and therefore, these have a limited impact on the provided rehabilitation dose. On the contrary, more complex devices, as exoskeletons, require a longer time to fit the robot for individual patients, and it is one of the main barriers to efficient use [26]. As in our approach, two studies on the same topic [8,9] used both robotic and non-robotic devices, supporting the idea that this approach can be effective and sustainable.

Anyway, robotic rehabilitation is not merely a matter of increasing the amount and intensity of therapy [27]. It provides extrinsic biofeedback that makes the patient aware of his movements and consequently allows him to modify his movement patterns, thus fostering his participation in the rehabilitation exercise [28-30]. Furthermore, it seems that the impact of technological rehabilitation on functional outcome could be optimized by offering more chances to the nervous system to experience "real" activity-related sensorimotor input during the training of upper limb movement [31].

From this knowledge and from our experience, it is very important to underline that physiotherapists have to perform an adequate training to acquire expertise with regard to these devices. With a correct use of them, the workload of the physiotherapists can be alleviated, their work time can be optimized, and they can focused toward personalizing rehabilitation treatments, according to each patient's residual abilities and progress in order to optimize functional recovery [27].

The organizational model herein discussed shows the feasibility of providing a comprehensive and a high technological level upper limb treatment that would be accessible to a high number of patients at once, while preserving an adequate training time and physiotherapist supervision. Finally, thanks to the sustainability in terms of optimizing human resources, this model can be applied to the usual care of the clinical practice considering the advantages that the robotic devices can provide (for example, to objectively measure the improvement during the therapy) $[32,33]$.

\section{Limitations of the Study}

This study was conducted in a highly specialized rehabilitation center where all physiotherapists had an expertise in robotic rehabilitation and used these devices for more than one year. Therefore, this could limit the extension of our results to different rehabilitation units with lower levels of expertise. However, we believe that this limitation does not severely impact on the application of the results, since a high level of expertise is required to efficiently use these devices. Moreover, this study was not aimed to evaluate the validity and the reliability of the used outcome measures. Finally, this study did not compare the effect of different physiotherapist/patient ratios on the outcome of the rehabilitation process. These aspects should be addressed in future studies.

\section{Conclusions}

In conclusion, this study showed the feasibility, in terms of rehabilitation dose and patients' satisfaction, of a new organizational model, where a group of patients supervised by one physiotherapist is treated with a set of robotic and sensor-based devices. Moreover, it highlighted that the mean disability of the group, as measured by the Barthel Index, could influence the proper physiotherapist/patient ratio. Future studies should be addressed to analyze the impact of this new model on the rehabilitation outcomes.

Supplementary Materials: The following are available online at http://www.mdpi.com/2076-3417/9/24/5357/s1.

Author Contributions: Conceptualization, I.A. and M.G.; methodology, I.A., M.G.; formal analysis, M.G.; investigation, C.P. and A.C.; data curation, C.P. and M.G.; writing-original draft preparation, I.A., S.L. and M.G.; writing-review and editing, I.A. and M.G.; supervision, I.A. and L.P. 
Funding: This research received no external funding.

Acknowledgments: The authors would like to thank Enrica Di Sipio for her help in making figures, Manuel Triolo for the support given from a linguistic standpoint and Claudia Loreti for administrative and technical support.

Conflicts of Interest: The authors declare no conflict of interest.

\section{References}

1. World Health Organization. International Classification of Functioning, Disability and Health: ICF; World Health Organization: Geneva, Switzerland, 2001.

2. Mehrholz, J.; Pohl, M.; Platz, T.; Kugler, J.; Elsner, B. Electromechanical and robot-assisted arm training for improving activities of daily living, arm function, and arm muscle strength after stroke. Cochrane Database Syst. Rev. 2015. [CrossRef]

3. Laut, J.; Porfiri, M.; Raghavan, P. The Present and Future of Robotic Technology in Rehabilitation. Curr. Phys. Med. Rehabil. Rep. 2016, 4, 312-319. [CrossRef]

4. Hayward, K.S.; Kramer, S.F.; Thijs, V.; Ratcliffe, J.; Ward, N.S.; Churilov, L.; Jolliffe, L.; Corbett, D.; Cloud, G.; Kaffenberger, T.; et al. A systematic review protocol of timing, efficacy and cost effectiveness of upper limb therapy for motor recovery post-stroke. Syst. Rev. 2019, 8, 187. [CrossRef] [PubMed]

5. Wagner, T.H.; Lo, A.C.; Peduzzi, P.; Bravata, D.M.; Huang, G.D.; Krebs, H.I.; Ringer, R.J.; Federman, D.G.; Richards, L.G.; Haselkorn, J.K.; et al. An economic analysis of robot-assisted therapy for long-term upper-limb impairment after stroke. Stroke 2011, 42, 2630-2632. [CrossRef] [PubMed]

6. Lo, K.; Stephenson, M.; Lockwood, C. The economic cost of robotic rehabilitation for adult stroke patients: A systematic review. JBI Database Syst. Rev. Implement. Rep. 2019, 17, 520-547. [CrossRef] [PubMed]

7. Masiero, S.; Poli, P.; Armani, M.; Ferlini, G.; Rizzello, R.; Rosati, G. Robotic upper limb rehabilitation after acute stroke by NeReBot: Evaluation of treatment costs. Biomed Res. Int. 2014, 2014. [CrossRef]

8. Atashzar, S.F.; Shahbazi, M.; Tavakoli, M.; Patel, R.V. A Computational-Model-Based Study of Supervised Haptics-Enabled Therapist-in-the-Loop Training for Upper-Limb Poststroke Robotic Rehabilitation. IEEE/ASME Trans. Mechatron. 2018, 23, 563-574. [CrossRef]

9. Buschfort, R.; Brocke, J.; Heß, A.; Werner, C.; Waldner, A.; Hesse, S. The arm studio to intensify the upper limb rehabilitation after stroke: Concept, acceptance, utilization and preliminary clinical results. J. Rehabil. Med. 2010, 42, 310-314. [CrossRef] [PubMed]

10. Hesse, S.; Heß, A.; Werner, C.C.; Kabbert, N.; Buschfort, R. Effect on arm function and cost of robot-assisted group therapy in subacute patients with stroke and a moderately to severely affected arm: A randomized controlled trial. Clin. Rehabil. 2014, 28, 637-647. [CrossRef]

11. Bustamante Valles, K.; Montes, S.; de Madrigal, M.J.; Burciaga, A.; Martínez, M.E.; Johnson, M.J. Technology-assisted stroke rehabilitation in Mexico: A pilot randomized trial comparing traditional therapy to circuit training in a Robot/technology-assisted therapy gym. J. Neuroeng. Rehabil. 2016, 13, 83. [CrossRef]

12. Aprile, I.; Cruciani, A.; Germanotta, M.; Gower, V.; Pecchioli, C.; Cattaneo, D.; Vannetti, F.; Padua, L.; Gramatica, F. Upper Limb Robotics in Rehabilitation: An Approach to Select the Devices, Based on Rehabilitation Aims, and Their Evaluation in a Feasibility Study. Appl. Sci. 2019, 9, 3920. [CrossRef]

13. Aprile, I.; Germanotta, M.; Cruciani, A.; Loreti, S.; Pecchioli, C.; Cecchi, F.; Montesano, A.; Galeri, S.; Diverio, M.; Falsini, C.; et al. Upper Limb Robotic Rehabilitation after Stroke: A Multicenter, Randomized Clinical Trial. J. Neurol. Phys. Ther. 2019, in press. [CrossRef]

14. Shah, S.; Vanclay, F.; Cooper, B. Improving the sensitivity of the Barthel Index for stroke rehabilitation. J. Clin. Epidemiol. 1989, 42, 703-709. [CrossRef]

15. Demeurisse, G.; Demol, O.; Robaye, E. Motor evaluation in vascular hemiplegia. Eur. Neurol. 1980, 19, 382-389. [CrossRef] [PubMed]

16. Korner-Bitensky, N.; Mayo, N.; Cabot, R.; Becker, R.; Coopersmith, H. Motor and functional recovery after stroke: Accuracy of physical therapists' predictions. Arch. Phys. Med. Rehabil. 1989, 70, 95-99. [PubMed]

17. Miller, M.D.; Paradis, C.F.; Houck, P.R.; Mazumdar, S.; Stack, J.A.; Rifai, A.H.; Mulsant, B.; Reynolds, C.F. Rating chronic medical illness burden in geropsychiatric practice and research: Application of the Cumulative Illness Rating Scale. Psychiatry Res. 1992, 41, 237-248. [CrossRef] 
18. Conwell, Y.; Forbes, N.T.; Cox, C.; Caine, E.D. Validation of a measure of physical illness burden at autopsy: The Cumulative Illness Rating Scale. J. Am. Geriatr. Soc. 1993, 41, 38-41. [CrossRef]

19. Salvi, F.; Miller, M.D.; Grilli, A.; Giorgi, R.; Towers, A.L.; Morichi, V.; Spazzafumo, L.; Mancinelli, L.; Espinosa, E.; Rappelli, A.; et al. A manual of guidelines to score the modified cumulative illness rating scale and its validation in acute hospitalized elderly patients. J. Am. Geriatr. Soc. 2008, 56, 1926-1931. [CrossRef]

20. Brokelman, R.B.G.; Haverkamp, D.; van Loon, C.; Hol, A.; van Kampen, A.; Veth, R. The validation of the visual analogue scale for patient satisfaction after total hip arthroplasty. Eur. Orthop. Traumatol. 2012, 3, 101-105. [CrossRef]

21. Kwakkel, G.; van Peppen, R.; Wagenaar, R.C.; Wood Dauphinee, S.; Richards, C.; Ashburn, A.; Miller, K.; Lincoln, N.; Partridge, C.; Wellwood, I.; et al. Effects of augmented exercise therapy time after stroke: A meta-analysis. Stroke 2004, 35, 2529-2539. [CrossRef]

22. Byl, N.N.; Pitsch, E.A.; Abrams, G.M. Functional outcomes can vary by dose: Learning-based sensorimotor training for patients stable poststroke. Neurorehabil. Neural Repair 2008, 22, 494-504. [CrossRef] [PubMed]

23. Langhorne, P.; Wagenaar, R.; Partridge, C. Physiotherapy after stroke: More is better? Physiother. Res. Int. 1996, 1, 75-88. [CrossRef] [PubMed]

24. Kwakkel, G. Impact of intensity of practice after stroke: Issues for consideration. Disabil. Rehabil. 2006, 28, 823-830. [CrossRef] [PubMed]

25. Huang, H.-C.; Chung, K.-C.; Lai, D.-C.; Sung, S.-F. The impact of timing and dose of rehabilitation delivery on functional recovery of stroke patients. J. Chin. Med. Assoc. 2009, 72, 257-264. [CrossRef]

26. Swank, C.; Sikka, S.; Driver, S.; Bennett, M.; Callender, L. Feasibility of integrating robotic exoskeleton gait training in inpatient rehabilitation. Disabil. Rehabil. Assist. Technol. 2019. [CrossRef]

27. Poli, P.; Morone, G.; Rosati, G.; Masiero, S. Robotic Technologies and Rehabilitation: New Tools for Stroke Patients' Therapy. Biomed Res. Int. 2013, 2013. [CrossRef]

28. van Vliet, P.M.; Wulf, G. Extrinsic feedback for motor learning after stroke: What is the evidence? Disabil. Rehabil. 2006, 28, 831-840. [CrossRef]

29. Masiero, S.; Poli, P.; Rosati, G.; Zanotto, D.; Iosa, M.; Paolucci, S.; Morone, G. The value of robotic systems in stroke rehabilitation. Expert Rev. Med. Devices 2014. [CrossRef]

30. Gassert, R.; Dietz, V. Rehabilitation robots for the treatment of sensorimotor deficits: A neurophysiological perspective. J. Neuroeng. Rehabil. 2018, 15, 46. [CrossRef]

31. Nudo, R.J. Functional and structural plasticity in motor cortex: Implications for stroke recovery. Phys. Med. Rehabil. Clin. N. Am. 2003, 14, S57-S76. [CrossRef]

32. Germanotta, M.; Vasco, G.; Petrarca, M.; Rossi, S.; Carniel, S.; Bertini, E.; Cappa, P.; Castelli, E. Robotic and clinical evaluation of upper limb motor performance in patients with Friedreich's Ataxia: An observational study. J. Neuroeng. Rehabil. 2015, 12, 41. [CrossRef] [PubMed]

33. Germanotta, M.; Cruciani, A.; Pecchioli, C.; Loreti, S.; Spedicato, A.; Meotti, M.; Mosca, R.; Speranza, G.; Cecchi, F.; Giannarelli, G.; et al. Reliability, validity and discriminant ability of the instrumental indices provided by a novel planar robotic device for upper limb rehabilitation. J. Neuroeng. Rehabil. 2018, 15, 39. [CrossRef] [PubMed]

(C) 2019 by the authors. Licensee MDPI, Basel, Switzerland. This article is an open access article distributed under the terms and conditions of the Creative Commons Attribution (CC BY) license (http://creativecommons.org/licenses/by/4.0/). 